\title{
Choice Disability as a target for non-medical HIV intervention
}

\author{
Rebecca de Boer \\ Department of Mathematics and Statistics \\ University of Ottawa \\ 585 King Edward Avenue \\ Ottawa, ON, K1N6N5 \\ Canada \\ and
}

Frithjof Lutscher*

Department of Mathematics and Statistics, and Department of Biology

University of Ottawa

585 King Edward Avenue

Ottawa, ON, K1N6N5

Canada

March 15, 2018

${ }^{*}$ Corresponding author 


\begin{abstract}
Even though medical intervention measures against HIV transmission are available, the epidemic continues to spread in several sub-Saharan African countries. Empirical studies indicate that many people are unable to implement prevention strategies because of individual factors, such as extreme poverty or lack of education, but also because or relational factors, such as gender-based violence or transactional sex. This phenomenon, known as choice disability, may be such a large obstacle in the effectiveness of medical interventions that several field trials of structural (non-medical) interventions are underway that address these issues. While dynamical-systems models are frequently used to advise management and policy around infectious diseases, they typically assume that individuals are free to make optimal choices. We derive and analyze a novel model where individuals have a certain choice status, based on which they are more or less likely to transmit or receive the infection. Choice status is affected by social interactions. When studying the model in the absence of an infectious disease, we find that structural interventions aimed at raising the status of one group can have the unintended side effect of lowering the status of another group. When combined with an epidemic model, we find that the same structural interventions can even increase the total prevalence of a disease in the population. Our model provides a framework to evaluate the possible effectiveness of structural intervention in an epidemic.
\end{abstract}

\title{
Keywords
}

HIV epidemic model; multigroup model; choice-disability; structural intervention; dynamical system

\section{Introduction}

Choice disability is a term mostly found in the literature on HIV and HIV prevention measures [2]. Research shows that in spite of the availability of medical interventions (e.g. condoms), some people are unable to make the choice to protect themselves from HIV. This inability may be due to individual factors (e.g. level of education) but more often than not is relational. For example, power gradients in personal relationships may prevent the ne-

gotiation of condom use. Several different factors have been described as 
so-called choice-disability risk-factors for HIV prevention [2]. These include: educational and economic disparities within relationships, food insecurity, intimate partner- and gender-based violence [3, 25] along with coerced [4], inter-generational [14, 27], and transactional sex [23]. There is a strong positive correlation between the number of choice-disability factors that an individual has accumulated and the risk of HIV infection [2]. All these studies indicate that knowledge and consideration of these factors can be crucial to the success of medical interventions. Even more, addressing these nonmedical aspects of HIV with social programs and other interventions could be an important step in its own right towards ending the HIV crises in large parts of Africa. Field trials are now underway to test the effectiveness of nonmedical interventions that target various choice-disability factors in lowering HIV infection risk [5, 13].

Dynamical systems models have a long and distinguished history in describing, understanding, and treating the HIV epidemic, including withinhost dynamics [31], between-host epidemics [1], multigroup models [30], and estimation of unreported cases [16]. The inclusion of choice-disability factors to structure the population is novel. Recently, we presented and analyzed a model that focused on non-relational factors of choice disability, such as extreme poverty [17]. We found that substantial declines in HIV prevalence are possible with carefully designed, non-medical interventions that focus on giving people choices with respect to HIV prevention. In that work, we did not address the relational aspect inherent in many aspects of choice disability, yet, many choice-disability factors arise only in the interaction between two individuals (as discussed previously). Here, we develop a mathematical model of HIV transmission in the context of relational choice disability and use it to consider the potential effects of non-medical HIV-prevention interventions targeting choice disability.

The disease transmission portion of the model is a multigroup SI model $[12,19]$ that structures the population by gender and multiple choice classes. For example, a choice class can correspond to the number of choice-disability risk-factors that an individual has accumulated. Individuals change choice class depending on individual and relational factors, as described above. While transition between choice classes due to individual factors is represented by simple linear rates, modelling choice disability due to relational factors involves contact terms that are superficially similar to those used for the disease transmission process. However, since choice disability can be acquired through contact with partners who are not themselves choice disabled, 
these transitions behave differently from a disease transmission model.

Section 2 provides a mathematical description of the complete model including both HIV and choice-disability elements. Section 3 provides some mathematical analysis, focusing on the choice-disability portion of the model. The simulation results in Section 4 focus on relational sources of choice disability and begin by considering only the choice-disability portion of the model. To evaluate the potential impact of non-medical interventions that target the choice-disability status of individuals, we turn to numerical simulations. In contrast to our previous model, where choice disability was based on individual factors and non-medical interventions were always positive, we here find that in the case of relational factors, the outcome of non-medical interventions depends subtly on the structure and relative strength of these relationships.

\section{Model}

To model HIV transmission in the context of choice disability, we structure the population by gender, HIV status, and choice status. Whereas gender and HIV status are binary properties, we formulate the model for any finite number of choice classes. For example, in our context, these choice classes could correspond to the number of choice-disability risk-factors that an individual has experienced in their life (see Introduction). It is known that the number of choice-disability risk-factors is positively correlated with HIV risk [2]. We denote these classes with index $i \in\{1, \ldots, n\}$, where higher numbers correspond to more factors. The model is summarized in the flow diagram in Figure 1. The equations for the $i$ th choice disability level are

$$
\begin{aligned}
\dot{S}_{i}^{F} & =\Lambda_{i}^{F}+\Psi_{i+1}^{M} S_{i+1}^{F}+\Gamma_{i-1}^{M} S_{i-1}^{F}-\Psi_{i}^{M} S_{i}^{F}-\Gamma_{i}^{M} S_{i}^{F}-B_{i}^{M} S_{i}^{F}-d_{i}^{S^{F}} S_{i}^{F} \\
\dot{I}_{i}^{F} & =\Psi_{i+1}^{M} I_{i+1}^{F}+\Gamma_{i-1}^{M} I_{i-1}^{F}+B_{i}^{M} S_{i}^{F}-\Psi_{i}^{M} I_{i}^{F}-\Gamma_{i}^{M} I_{i}^{F}-d_{i}^{I^{F}} I_{i}^{F} \\
\dot{S}_{i}^{M} & =\Lambda_{i}^{M}+\Psi_{i+1}^{F} S_{i+1}^{M}+\Gamma_{i-1}^{F} S_{i-1}^{M}-\Psi_{i}^{F} S_{i}^{M}-\Gamma_{i}^{F} S_{i}^{M}-B_{i}^{F} S_{i}^{M}-d_{i}^{S^{M}} S_{i}^{M} \\
\dot{I}_{i}^{M} & =\Psi_{i+1}^{F} I_{i+1}^{M}+\Gamma_{i-1}^{F} I_{i-1}^{M}+B_{i}^{F} S_{i}^{M}-\Psi_{i}^{F} I_{i}^{M}-\Gamma_{i}^{F} I_{i}^{M}-d_{i}^{I^{M}} I_{i}^{M}
\end{aligned}
$$

where $\Psi_{i}^{\bullet}, \Gamma_{i}^{\bullet}$ and $B_{i}^{\bullet}$ are functions of the populations with gender $\bullet$.

Parameters $\Lambda_{i}^{\bullet}$ and $d_{i}^{S^{\bullet}}, d_{i}^{I^{\bullet}}$ are the rates of recruitment into the system (i.e. the incoming cohort of sexually active people) and removal from the system (i.e. through ceasing sexual activity or death), respectively. Individuals move between different compartments either due to a change in choice class 


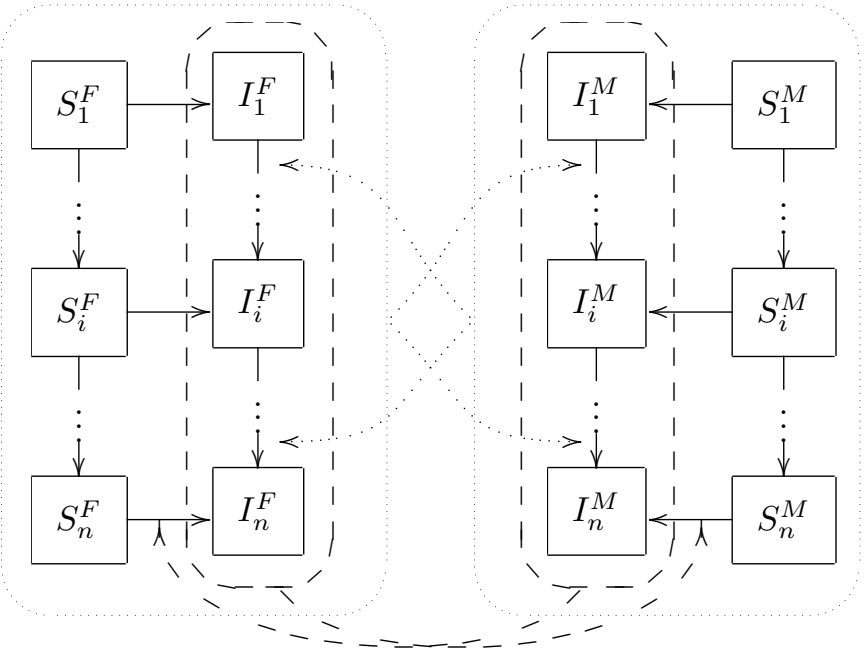

Figure 1: Flow diagram for model (1). The female population (left dotted box) and male population (right dotted box) are each subdivided into choice classes (vertical) and infection state (horizontal) with infectious compartments combined in dashed boxes. Horizontal arrows indicate infection (modeled by functions $B_{i}^{\bullet}$ ) depending on contact with the infectious individuals from the other sex (dashed arrows). Vertical arrows indicate acquisition of (down, $\Gamma_{i}^{\bullet}$ ) and recovery from $\left(u p, \Psi_{i}^{\bullet}\right.$ ) choice disability that can result from contact with individuals from any compartment of the other sex (dotted arrows). 
or through infection. Functions $\Gamma_{i}^{\bullet}$ and $\Psi_{i}^{\bullet}$ describe the per-capita rates of choice disability acquisition (i.e. moving from level $i$ to $i+1$ ) and recovery (i.e. moving from level $i$ to $i-1$ ), respectively. Disease transmission is captured by functions $B_{i}^{\bullet}$. We describe the functional forms of these three aspects of our model in detail below. All parameters are non-negative. Notation and interpretation are summarized in Table 1.

The rates of moving between choice classes incorporate individual factors as linear rates and relational aspects as bilinear terms. These rates depend on the number of individuals in each choice class and are given by

$$
\begin{aligned}
& \Gamma_{i}^{\bullet}=\lambda_{i}^{\bullet}+\sum_{j=1}^{n} \gamma_{i j}^{\bullet} N_{j}^{\bullet} \\
& \Psi_{i}^{\bullet}=\mu_{i}^{\bullet}+\sum_{j=1}^{n} \psi_{i j}^{\bullet} N_{j}^{\bullet}
\end{aligned}
$$

where $N_{j}^{\bullet}=S_{j}^{\bullet}+I_{j}^{\bullet}$. Parameters $\lambda_{i}^{\bullet}$ and $\mu_{i}^{\bullet}$ stand for the rates due to individual factors whereas $\gamma_{i j}^{\bullet}$ and $\psi_{i j}^{\bullet}$ encode the contact structure between individuals from various choice classes. For example, $\gamma_{i j} N_{j}$ is the rate at which an individual in choice class $i$ acquires an additional choice disability factor through interactions with individuals of choice class $j$. Our exploration of effects and side-effects of non-medical interventions in Section 4 will focus on the parameters $\gamma_{i j}$.

There are only finitely many possible choice levels in our model, and individuals cannot leave the population via changes in choice status. Since one cannot move up from level 1 nor down from level $n$, we impose the conditions $\Psi_{1}^{\bullet}=0$ and $\Gamma_{n}^{\bullet}=0$.

Functions $B_{i}^{\bullet}$ describe the rate of disease transmission via bilinear transmission terms. They depend on the number of infected individuals in each choice class and are given by

$$
B_{i}^{\bullet}=\sum_{j=1}^{n} \beta_{i j}^{\bullet} I_{j}^{\bullet} .
$$

This formulation satisfies all the balance requirements for multigroup models $[12]$.

Formally, the summations in (2) and (3) look similar. However, the infection process can only move an individual to an infectious class if the contact 
is with an infectious person. The choice-disability process can move individuals up or down in choice-disability level independent of the state of the person with whom the contact is. This difference, as simple and obvious as it may seem, has major ramifications for the analysis of the model. In particular, a 'disease-free' state is not necessarily 'simple' in that the population can still be distributed across all choice-disability levels. Several of the classical analytical approaches to analyze model behavior fail, in particular, the application of standard Liapunov functions [29] is impossible. Nonetheless, we can gain some insights into the analytical properties of our model in the next section.

As a word of caution, we want to point out a few difficulties that we will elaborate further in the discussion. Different choice-disability factors have different causes and effects. For example, a certain level of income might be considered low, but whether this leads to income disparity between partners depends on the partner. And what might be a relatively higher income with one could be a relatively lower income with another. The same is true for education. However, while a relationship with an extremely poor person can hurt a relatively richer partner financially long after the relationship is over, the same is probably not true for education levels. It is, however, certainly true that a person who experienced sexual violence in one partnership will carry this experience forward to future relationships. All this is to say that our model, while already quite complex, may still be too coarse to pick up all details. However, we believe that it is a useful model to examine various effects (as we will see in Section 4) and that it would probably impossible to write down and parametrize a model in which each choice-disability factor is considered separately.

\section{Analysis}

Equations (1) define a system of ordinary differential equations on $\mathbb{R}^{4 n}$. In this generality, specific analytical results are difficult to come by. We prove that the system has globally existing, bounded solutions. Then we specialize to the simplest possible interesting case of two choice classes, and we prove uniqueness and global stability of the steady state.

Lemma 1. All solutions to equations (1) with non-negative initial conditions remain non-negative, exist for all times, and will eventually enter the feasible 


\begin{tabular}{ll} 
Parameter & Description \\
\hline$\Lambda_{i}^{\bullet}$ & Recruitment rate for choice level $i$ \\
$d_{i}^{\boldsymbol{S}^{\bullet}}, d_{i}^{\boldsymbol{\bullet}^{\bullet}}$ & Per capita removal rates for choice level $i$ \\
$\lambda_{i}^{\bullet}$ & $\begin{array}{l}\text { Per capita disability acquisition rate due to individual factors } \\
\text { for choice level } i\end{array}$ \\
$\gamma_{i j}^{\bullet}$ & $\begin{array}{l}\text { Effective contact rate with choice level } j \text { for disability acqui- } \\
\text { sition by individuals in choice level } i\end{array}$ \\
$\mu_{i}^{\bullet}$ & $\begin{array}{l}\text { Per capita disability recovery rate due to individual factors } \\
\text { for choice level } i\end{array}$ \\
$\psi_{i j}^{\bullet}$ & $\begin{array}{l}\text { Effective contact rate with choice level } j \text { for disability recovery } \\
\text { by individuals in choice level } i\end{array}$ \\
$\beta_{i j}^{\bullet}$ & Effective contact rate with choice level $j$ for disease transmis- \\
\hline
\end{tabular}

Table 1: Summary and descriptions of parameters for the model in (1) 
region

$D=\left\{\left(S_{1}^{F}, I_{1}^{F}, S_{1}^{M}, I_{1}^{M}, \ldots, S_{n}^{F}, I_{n}^{F}, S_{n}^{M}, I_{n}^{M}\right) \in \mathbb{R}_{+}^{4 n} \mid N_{T o t}^{F} \leq \frac{\Lambda^{F}}{d^{F}}, N_{T o t}^{M} \leq \frac{\Lambda^{M}}{d^{M}}\right\}$,

where $N_{\text {Tot }}^{\bullet}=\sum_{i=1}^{n} S_{i}^{\bullet}+I_{i}^{\bullet}$.

Proof. The vector field is smooth, and therefore local existence and uniqueness of solutions is guaranteed by standard theory [21]. If one of the susceptible populations is zero, i.e. $S_{i}^{\bullet}=0$, then $\dot{S}_{i}^{\bullet} \geq 0$. Similarly, if one of the infectious populations is zero, i.e. $I_{i}^{\bullet}=0$, then $\dot{I}_{i}^{\bullet} \geq 0$. Hence, solutions to non-negative initial conditions remain non-negative and therefore the positive orthant, $\mathbb{R}_{+}^{4 n}$ is invariant. Adding all the equations for each sex, gives

$$
\dot{N}_{T o t}^{\bullet}=\sum_{i=1}^{n} \Lambda_{i}^{\bullet}-d_{i}^{S^{\bullet}} S_{i}^{\bullet}-d_{i}^{I^{\bullet}} I_{i}^{\bullet} \leq \Lambda^{\bullet}-d^{\bullet} N_{T o t}^{\bullet}
$$

where $d^{\bullet}=\min \left(d_{i}^{I^{\bullet}}, d_{i}^{S^{\bullet}}\right), \Lambda^{\bullet}=\sum_{i=1}^{n} \Lambda_{i}^{\bullet}$. These inequalities imply that the region $D$ is invariant and that all non-negative solutions eventually enter this region. The existence of a bounded, invariant attracting region implies global existence of solutions [21].

Choice disability acquisition and recovery in model (1) do not depend on infection status. If any differences in the removal rates $d_{i}^{S^{\bullet}}$ and $d_{i}^{I^{\bullet}}$ are neglected and both removal rates are assumed to be equal to $d_{i}^{\bullet}$, then the model can be collapsed to consider choice disability separately from HIV infection. Adding the equations for $S_{i}^{\bullet}$ and $I_{i}^{\bullet}$, we obtain the model

$$
\begin{aligned}
& \dot{N}_{i}^{F}=\Lambda_{i}^{F}+\Psi_{i+1}^{M} N_{i+1}^{F}+\Gamma_{i-1}^{M} N_{i-1}^{F}-\Psi_{i}^{M} N_{i}^{F}-\Gamma_{i}^{M} N_{i}^{F}-d_{i}^{F} N_{i}^{F}, \\
& \dot{N}_{i}^{M}=\Lambda_{i}^{M}+\Psi_{i+1}^{F} N_{i+1}^{M}+\Gamma_{i-1}^{F} N_{i-1}^{M}-\Psi_{i}^{F} N_{i}^{M}-\Gamma_{i}^{F} N_{i}^{M}-d_{i}^{M} N_{i}^{M},
\end{aligned}
$$

that tracks the changes in choice-disability status only. This 'choice-disability model' has phase space $\mathbb{R}_{+}^{2 n}$ and is, in the simplest, non-trivial case $(n=2)$, amenable to some more analysis. Specifically, we will prove that this model has a unique, globally stable steady state in the feasible region. In the next section, we will then analyze how this steady state depends on parameters, and we will find some surprising insights about secondary and tertiary effects of interventions that aim to raise the status of one sex. 
Theorem 2. Consider the 'choice-disability model' in (4) for $n=2$. Assume that all parameter values are positive and that the removal rate is independent of social status (i.e. $d_{i}^{\bullet}=d^{\bullet}>0$ ). Then the sytem has a unique steady state in the feasible region, and this state is globally asymptotically stable.

Please note that the theorem holds under weaker conditions, where some of the parameters are allowed to be zero. Clearly, the removal rates $d^{\bullet}$ and at least some of the recruitment rates $\Lambda_{i}^{\bullet}$ must be positive, but, for example, the theorem still holds if $\psi_{i j}^{\bullet}=0$.

Proof. It is convenient to use the total population for each sex rather than the first social status. That is, we replace equations (4) by the equivalent system

$$
\begin{aligned}
\dot{N}_{\text {Tot }}^{F} & =\Lambda^{F}-d^{F} N_{\text {Tot }}^{F}, \\
\dot{N}_{2}^{F} & =\Lambda_{2}^{F}+\Gamma_{1}^{M}\left(N^{M}\right)\left(N_{\text {Tot }}^{F}-N_{2}^{F}\right)-\left(\Psi_{2}^{M}\left(N^{M}\right)+d^{F}\right) N_{2}^{F}, \\
\dot{N}_{\text {Tot }}^{M} & =\Lambda^{M}-d^{M} N_{\text {Tot }}^{M}, \\
\dot{N}_{2}^{M} & =\Lambda_{2}^{M}+\Gamma_{1}^{F}\left(N^{F}\right)\left(N_{\text {Tot }}^{M}-N_{2}^{M}\right)-\left(\Psi_{2}^{F}\left(N^{F}\right)+d^{M}\right) N_{2}^{M},
\end{aligned}
$$

where $\Lambda^{\bullet}=\Lambda_{1}^{\bullet}+\Lambda_{2}^{\bullet}$ as before.

The first and third equation decouple, and the solutions approach the respective steady states $N_{\text {Tot }}^{\bullet} \rightarrow \Lambda^{\bullet} / d^{\bullet}$. Hence, the hyperplane defined by these steady states is invariant and attractive. We can study the remaining two equations on this hyperplane.

We write the equations for $x=N_{2}^{F}$ and $y=N_{2}^{M}$ as $\dot{x}=f(x, y)$ and $\dot{y}=g(x, y)$ with

$$
f(x, y)=\Lambda_{2}^{F}-\left(A_{1}+\psi y\right) x+\left(A_{2}+\gamma y\right)\left(A_{3}-x\right),
$$

where $\psi=\psi_{12}^{M}-\psi_{11}^{M}, \gamma=\gamma_{12}^{M}-\gamma_{11}^{M}$, and

$$
\begin{aligned}
& A_{1}=\mu_{1}^{M}+d^{F}+\psi_{11}^{M} \frac{\Lambda^{M}}{d^{M}}, \\
& A_{2}=\lambda_{1}^{M}+\gamma_{11}^{M} \frac{\Lambda^{M}}{d^{M}}, \quad A_{3}=\Lambda^{F} / d^{F} .
\end{aligned}
$$

The equation for $g$ has a similar expression; we present details only for $f$.

Function $f$ is linear in $x$. For each fixed $y \in\left[0, \Lambda^{M}\right]$, we calculate

$$
f(0, y)>0 \text { and } f\left(\Lambda^{F} / d^{F}, y\right)<0 .
$$


In particular, for each $y$ there is a unique $x=G(y)$ so that $f(G(y), y)=0$. We calculate

$$
G(y)=\frac{\Lambda_{2}^{F}+A_{3}\left(A_{2}+\gamma y\right)}{A_{1}+A_{2}+(\psi+\gamma) y}
$$

Next, we find the derivative of $G$ as

$$
G^{\prime}(y)=\frac{C}{\left(A_{1}+A_{2}+(\psi+\gamma) y\right)^{2}},
$$

for some constant $C$. In particular, depending on the sign of $C$, we see that either, $G^{\prime}$ is positive and decreasing or negative and increasing. Accordingly, $G$ is either increasing and concave down or decreasing and concave up. In either case, $G$ is invertible, and the inverse, $y=G^{-1}(x)$, defines the $x$ nullcline in the $(x, y)$-plane.

The results for the $y$-nullcline are even simpler. For each $x \in\left[0, \Lambda^{F}\right]$, we have $g(x, 0)>0$ and $g\left(x, \Lambda^{M} / d^{M}\right)<0$. Since $g$ is linear in $y$, there is a unique $y=\widetilde{G}(x)$ with $g(x, \widetilde{G}(x))=0$. Function $\widetilde{G}$ has the same general form as $G$ above, and the set $y=\widetilde{G}(x)$ denotes the $y$-nullcline in the $(x, y)$-plane.

In the $(x, y)$-plane, the vectorfield $(f, g)$ points inwards along the boundary of the rectangle $\left[0, \Lambda^{F} / d^{F}\right] \times\left[0, \Lambda^{M} / d^{M}\right]$, which is therefore positively invariant. The $x$-nullcline is a curve that connects the line segment $\left[0, \Lambda^{F} / d^{F}\right] \times$ $\{0\}$ with the segment $\left[0, \Lambda^{F} / d^{F}\right] \times\left\{\Lambda^{M} / d^{M}\right\}$ inside the rectangle. The $y$ nullcline is a curve that connects the line segment $\{0\} \times\left[0, \Lambda^{M} / d^{M}\right]$ with the segment $\left\{\Lambda^{M} / d^{M}\right\} \times\left[0, \Lambda^{M} / d^{M}\right]$ inside the rectangle $\left[0, \Lambda^{F} / d^{F}\right] \times\left[0, \Lambda^{M} / d^{M}\right]$. Because these curves are continuous, they have to intersect at least once, and the number of intersection points has to be odd. If we substitute the expression for $x=G(y)$ into the function $g$, we obtain a quadratic equation for $y$. Hence, there can be at most two intersection points. Therefore, there must be exactly one in the feasible rectangle.

Finally, we use the Bendixon-Dulac criterion to exclude a periodic orbit. We multiply the vectorfield by $(x y)^{-1}$. We calculate

$$
\frac{f(x, y)}{x y}=\frac{\Lambda_{2}^{F}+A_{2} A_{3}}{x y}-\frac{A_{1}+A_{2}}{y}-(\psi-\gamma)+\frac{A_{3} \gamma}{x}
$$

and

$$
\frac{\partial}{\partial x}\left(\frac{f(x, y)}{x y}\right)=-\frac{1}{x^{2} y}\left(\Lambda_{2}^{F}+A_{3}\left(A_{2}+\gamma y\right)\right)
$$


Substituting the expression for $A_{2}$ and $\gamma$, we find

$$
A_{2}+\gamma y=\lambda_{1}^{M}+\gamma_{11}^{M} \frac{\Lambda^{M}}{d^{M}}+\left(\gamma_{12}^{M}-\gamma_{11}^{M}\right) y
$$

Since $0<y<\Lambda^{M} / d^{M}$, this expression is positive. Hence the expression in (10) is negative. Similar calculations show that the derivative of $g(x, y) /(x y)$ with respect to $y$ is negative inside the feasible rectangle. Hence, by the Bendixon-Dulac criterion, there cannot be a periodic orbit inside the rectangle.

For later reference, we give the explicit expressions for the steady state that we will study in subsequent chapters. In addition to the assumptions in the theorem, we assume that there is no recruitment directly into the second social status (i.e. $\Lambda_{2}^{\bullet}=0$ ) and that one of the following conditions holds:

$$
\gamma_{11}^{M}>0 \quad \text { or } \quad \lambda_{1}^{M}>0,
$$

i.e., that (female) individuals have a positive probability to acquire choice disability. The calculations are tedious but not complicated. We find the steady state values as

$$
\begin{aligned}
& N_{\text {Tot }}^{M *}=\frac{\Lambda_{1}^{M}}{d^{M}}, \quad N_{2}^{M^{*}}=\frac{\frac{\Lambda_{1}^{M}}{d^{M}} \Gamma_{1}^{F}\left(N^{F^{*}}\right)}{\Gamma_{1}^{F}\left(N^{F^{*}}\right)+\Psi_{2}^{F}\left(N^{F^{*}}\right)+d^{M}}, \\
& N_{\text {Tot }}^{F}=\frac{\Lambda_{1}^{F}}{d^{F}}, \quad N_{2}^{F^{*}}=\frac{-b \pm \sqrt{b^{2}-4 a c}}{2 a}
\end{aligned}
$$

with coefficients

$$
\begin{aligned}
a= & \left(\frac{\Lambda_{1}^{M}}{d^{M}}\left(\gamma_{12}^{M}+\psi_{22}^{M}\right)+\lambda_{1}^{M}+\mu_{2}^{M}+d^{F}\right)\left(\gamma_{11}^{F}-\gamma_{12}^{F}\right) \\
& +\left(\frac{\Lambda_{1}^{M}}{d^{M}}\left(\gamma_{11}^{M}+\psi_{21}^{M}\right)+\lambda_{1}^{M}+\mu_{2}^{M}+d^{F}\right)\left(\psi_{21}^{F}-\psi_{22}^{F}\right) \\
b= & -\frac{\Lambda_{1}^{F}}{d^{F}} a-\frac{d^{F}}{\Lambda_{1}^{F}} c-h \\
c= & \frac{\Lambda_{1}^{F}}{d^{F}}\left[\left(\gamma_{11}^{M} \frac{\Lambda_{1}^{M}}{d^{M}}+\lambda_{1}^{M}\right)\left(\psi_{21}^{F} \frac{\Lambda_{1}^{F}}{d_{F}}+\mu_{2}^{F}+d^{M}\right)\right. \\
& \left.+\left(\gamma_{12}^{M} \frac{\Lambda_{1}^{M}}{d_{M}}+\lambda_{1}^{M}\right)\left(\gamma_{11}^{F} \frac{\Lambda_{1}^{F}}{d^{F}}+\lambda_{1}^{F}\right)\right]
\end{aligned}
$$


where

$$
\begin{aligned}
h= & \left(\psi_{21}^{M} \frac{\Lambda_{1}^{M}}{d^{M}}+\mu_{2}^{M}+d^{F}\right)\left(\psi_{22}^{F} \frac{\Lambda_{1}^{F}}{d^{F}}+\mu_{2}^{F}+d^{M}\right) \\
& +\left(\psi_{22}^{M} \frac{\Lambda_{1}^{M}}{d^{M}}+\mu_{2}^{M}+d^{F}\right)\left(\gamma_{12}^{F} \frac{\Lambda_{1}^{F}}{d^{F}}+\lambda_{1}^{F}\right) .
\end{aligned}
$$

It is possible, but again tedious, to show directly from these expressions that exactly one of the expressions for $N_{2}^{F^{*}}$ in (12) is in the feasible region.

\section{Qualitative effects of intervention}

We now investigate the qualitative behaviour of the model. We focus on the question of what effects a non-medical intervention, aimed at reducing choice disability, can have for HIV incidence and prevalence.

In the best possible scenario, the reproductive number, $R_{0}$, of $\mathrm{HIV}$ in a population of completely choice-enabled individuals would be less than unity and the disease could be eliminated. Then we would calculate $R_{0}$ in a population with choice-disability classes and estimate the required strength of intervention to push it below unity. We can use the next-generation method [34] to write a formula for $R_{0}$ but the expression is not particularly helpful since we need to evaluate it numerically.

Unfortunately, this best possible scenario seems too far out of reach at the moment in countries of southern Africa where HIV prevalence ranges from $9.1 \%$ in Malawi to $28.8 \%$ in Swaziland [32]. For that reason, we focus on the question of how much of a difference non-medical interventions, targeting the choice status of individuals, can make in the fight against HIV. Specifically, we look at the absolute or relative changes in HIV prevalence in relation to contact patterns and intervention strength of choice disability.

We limit our investigation of these questions to several, strategically chosen scenarios. We consider only the two levels of choice enabled ('high status') and choice disabled ('low status') without further subdividing levels of choice disability. We are particularly interested in relational aspects of choice disability and hence assume that transition to disability is caused solely by contacts such as sexual assault or inter-partner violence. We set $\lambda_{i}^{\bullet}=0$ and use

$$
\Gamma_{i}^{\bullet}=\sum_{j=1}^{n} \gamma_{i j}^{\bullet} N_{j}^{\bullet}
$$


We recently considered the opposite scenario, where the transition to choice disability was entirely based on individual factors (i.e. $\lambda_{i}^{\bullet}>0$ and $\gamma_{i j}^{\bullet}=0$ ) $[17]$.

Finally, we consider the case that choice-disabled individuals can only return to choice-enabled status though an intervention that does not depend on social contact. Hence, we set $\psi_{i j}^{\bullet}=0$ and use $\Psi_{i}^{\bullet}=\mu_{i}^{\bullet}$, where $\mu_{2}^{M} \geq 0$ denotes the strength of the intervention for choice-disabled women and $\mu_{2}^{F} \geq$ 0 denotes the strength of the intervention for choice-disabled men.

We use numerical simulations of the model to investigate how the possible outcomes of interventions depend on parameter values. Data are available for some aspects of the model, in particular for the population dynamics and for disease transmission rates [17], but parameter estimates for other aspects are quite uncertain (e.g. the disease-related contact rates). Data needed to estimate parameter values related to acquisition and recovery of choice disability are not yet available but are starting to be collected in controlled, randomized field trials [5]. For that reason, we choose parameter values to illustrate certain strategic scenarios and mechanisms and not with the intention of being directly applicable. Using the model, we identify cases and mechanisms in which well-meant interventions can have unintended negative consequences and we describe the scope of those consequences.

\subsection{Intervention and choice disability}

We begin by concentrating on the 'choice-disability model' (see Section 3), i.e. we neglect HIV infection for the moment.

We consider an intervention that targets choice-disabled women only, for example through education, and acts to enable them to make choices, for example regarding HIV prevention. This intervention is modelled by setting $\mu_{2}^{M}>0$ and $\mu_{2}^{F}=0$. While such an intervention has no direct effects on men, it will have an indirect impact on men through their contacts with women. There are two possible cases:

1. If choice-enabled men are more likely to become choice-disabled by contact with a choice-disabled woman than a choice-enabled woman (that is, if $\gamma_{11}^{F}<\gamma_{12}^{F}$ ), then the intervention for women will also benefit men allowing more of them to remain choice enabled. Therefore, we will call this case 'positive feedback'. This scenario could arise in the case of great disparity of education and/or income where the lack of 

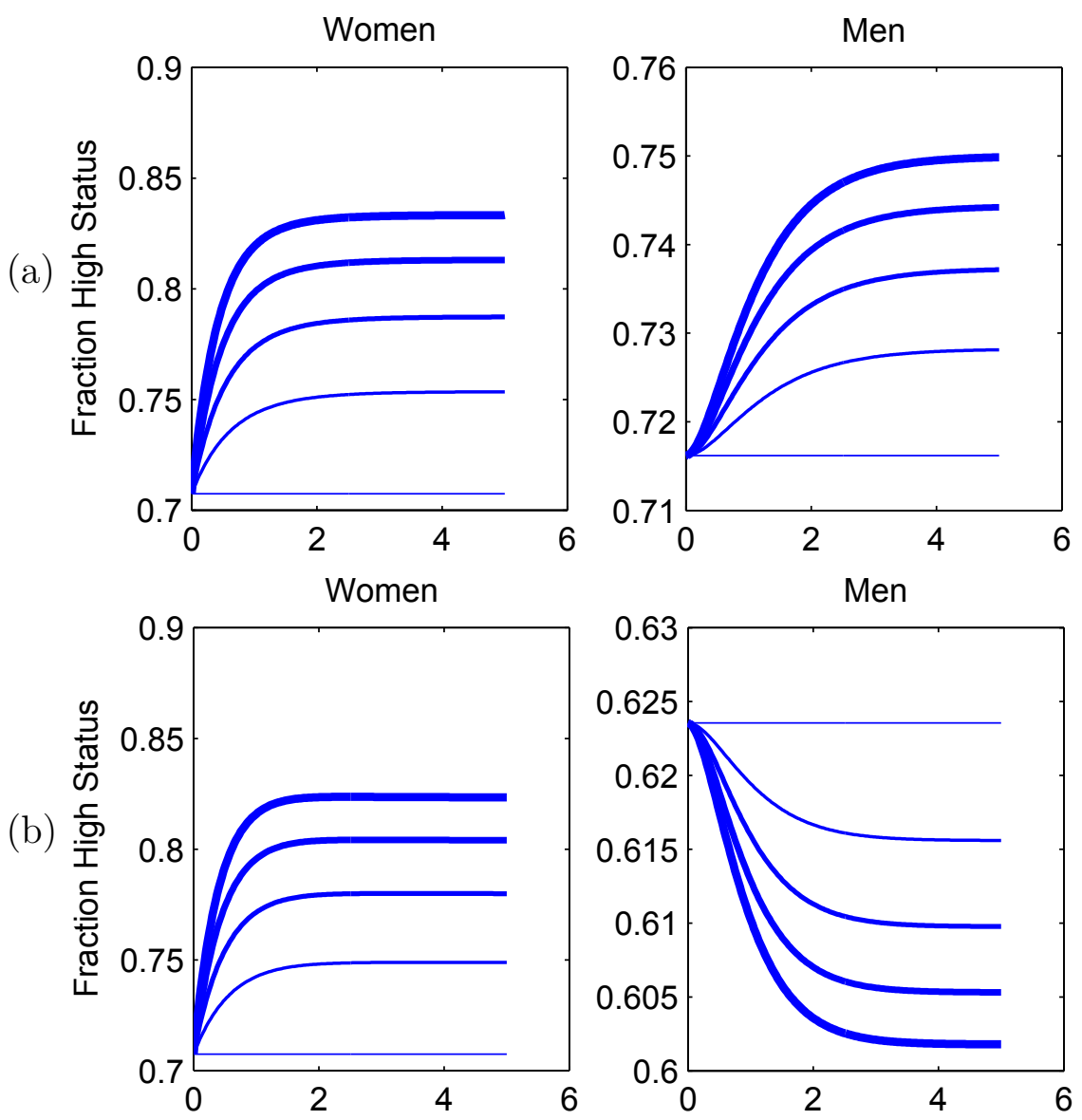

Figure 2: Changes in high status (i.e., choice enabled) men and women over time since implementation of an intervention to raise the choice status of women $\left(\mu_{2}^{M}>0\right)$. In (a) $\gamma_{11}^{F}<\gamma_{12}^{F}$ and the number of high-status men increases as the number of high-status women increases. This simulation uses contact parameters $\gamma_{11}^{F}=0.25, \gamma_{12}^{F}=0.75, \gamma_{11}^{M}=0.3$, and $\gamma_{12}^{M}=0.7$. In (b) $\gamma_{11}^{F}>\gamma_{12}^{F}$ and the number of high-status men decreases as the number of high-status women increases. This simulation uses contact parameters $\gamma_{11}^{F}=0.75, \gamma_{12}^{F}=0.25, \gamma_{11}^{M}=0.15$, and $\gamma_{12}^{M}=0.85$. For both cases, intervention parameter $\mu_{2}^{M}$ takes values $0,0.25,0.5,0.75,1$, with thicker lines corresponding to stronger interventions. The remaining parameters here and for all simulation results, unless otherwise noted, are $\Lambda_{1}^{F}=\Lambda_{1}^{M}=1$ and $d_{1}^{F}=d_{2}^{F}=d_{1}^{M}=d_{2}^{M}=1$. All parameters that are not explicitly listed are set to zero. 
resources in one partner (here the woman) limits the choices of the other (here the man).

2. If choice-enabled men are more likely to become choice-disabled by contact with a choice-enabled woman than a choice-disabled woman (that is, if $\gamma_{11}^{F}>\gamma_{12}^{F}$ ), then the intervention will be detrimental to men and more of them will become choice disabled. Accordingly, we will call this case 'negative feedback'. This scenario could arise in the context of transactional sex and/or income and education disparity, where the abundance of resources of one partner (here the woman) limits the choices of the other partner (here the man).

The effects and side effects from these two possibilities are illustrated in Figure 2. This figure shows how the fractions of men and women with high status (i.e. those who are choice enabled) evolve over time after an intervention measure is put in place. We choose initial conditions at the steady-state value in the absence of intervention and run simulations for several values of intervention strength $\mu_{2}^{M}$. The thin constant line indicates the steady state in the absence of intervention, thicker lines indicate larger values of $\mu_{2}^{M}$.

Parameters are chosen so that the results from Section 3 apply, i.e., we know that there is a unique, globally stable steady state for any level of intervention. In all cases, the fractions start at the state without intervention and monotonically approach the steady state with intervention. While the fraction of high-status women increases for any strength of intervention, the fraction of high-status men increases in the first case (top row of plots) and decreases in the second (bottom row).

Next, we illustrate how the outcome of an intervention depends on the contact parameters. We consider the equilibria $N_{1}^{\bullet *}$ and $N_{1}^{\bullet *}$ corresponding to the situation with and without intervention, respectively. We calculate the relative change in the number of high status individuals, as measured by

$$
R_{N_{1}}=\frac{N_{1 I}^{\bullet *}-N_{1}^{\bullet *}}{N_{1}^{\bullet *}} .
$$

In particular, if $R_{N_{i}}>0$ then the intervention has the (desired) outcome of increasing the number of high-status individuals in compartment $N_{1}^{\bullet}$.

We choose contact rates $\gamma_{11}^{F}$ and $\gamma_{11}^{M}$ in the range $[0,3]$ and keep the total contact rate per gender constant by setting $\gamma_{12}^{F, M}=3-\gamma_{11}^{F, M}$. The 
relative changes for women, men, and the whole choice-enabled population $\left(N_{1}^{F}+N_{1}^{M}\right)$ are displayed in the contour plots in Figure 3. The dashed cross in each plot indicates the lines $\gamma_{11}^{\bullet}=1.5=\gamma_{12}^{\bullet}$ where the rate of status change is independent of the state of the relating partner.

The first plot in Figure 3 reveals that women always benefit from an intervention aimed at only women, in the sense that $R_{N_{1}^{F}}>0$. It is also clear that the size of the beneficial effect depends on the contact parameters. It is particularly strong when $\gamma_{11}^{\bullet}$ are small, i.e. when the lowest risk of becoming choice enabled results from contacts with choice-enabled partners.

To explain this observation, we look at the numbers for men (second plot in Figure 3). When $\gamma_{11}^{F}=1.5=\gamma_{12}^{F}$ the intervention has no impact on men because interactions with high- and low-status women have the same effect on men. Therefore, men do not benefit when the status of women is raised. For values $\gamma_{11}^{F}>1.5>\gamma_{12}^{F}$, the intervention has a negative impact on men, reducing the number of high-status men in comparison to the no-intervention case. This reduction occurs because, with these contact parameters, highstatus women are more likely to harm the status of men than their lowstatus counterparts and the intervention increases the number of high-status women. Conversely, when $\gamma_{11}^{F}<1.5<\gamma_{12}^{F}$ the intervention benefits both, men and women.

With this information, we look again at the first plot for women where we observe additional feedback effects that were difficult or impossible to see in Figure 2. We consider the point in the center where $\gamma_{i j}^{\bullet}=1.5$ as a reference point where contact structure does not affect the rate of status change. Then the effect on women's status is increased in the direction of the first and third quadrants where either $\gamma_{11}^{\bullet}$ are both low or both high and decreased in the other two directions where one of $\gamma_{11}^{\bullet}$ is high and one is low.

When both $\gamma_{11}^{M, F}$ are low, the benefit to men increases the impact of the original intervention on women. However when $\gamma_{11}^{M}<1.5$, and $\gamma_{11}^{F}>1.5$ the harm to men also has a feedback effect, in this case weakening the original intervention on women. Similar strengthening and weakening effects are seen when $\gamma_{11}^{M}>1.5$. Despite the fact that an intervention benefiting women may harm men for some parameter combinations, the overall effect in the population is always positive in these simulations, as indicated in the final panel in Figure 3. In the next section, we explore how interventions for choice disability affect HIV incidence and prevalence. In particular, we are interested in whether the negative side effects that are possible from choicedisability interventions can lead to negative side effects for HIV as well. 

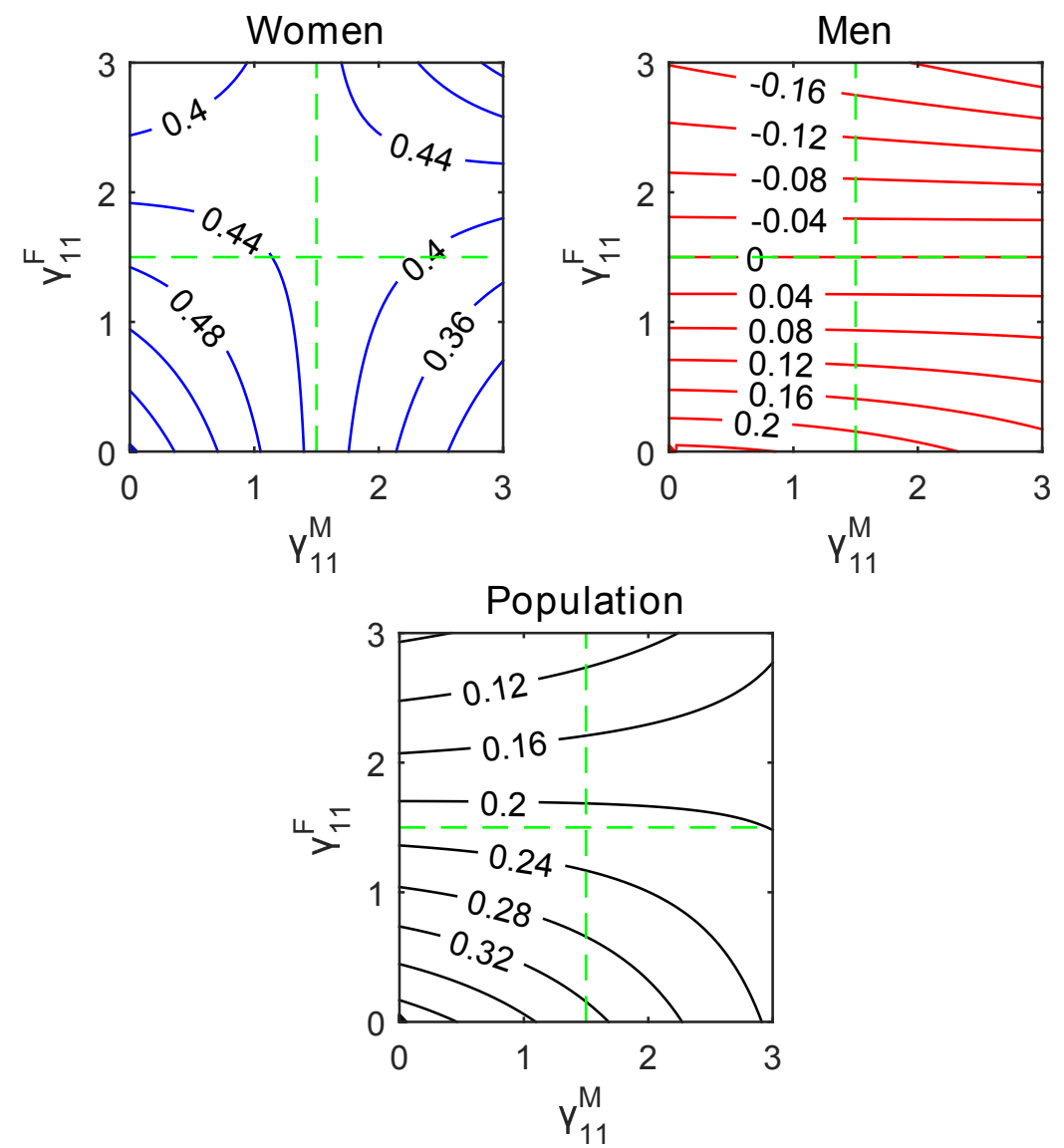

Figure 3: Contour plots showing the relative increase or decrease in the number of high-status women (top left), men (top right) and total population (bottom panel) when an intervention $\left(\mu_{2}^{M}=1\right)$ is implemented to raise the status of women. The fraction of high-status men decreases from the intervention if $\gamma_{11}^{F}>\gamma_{12}^{F}$, which happens when $\gamma_{11}^{F}>1.5$ since the total contact rates are held constant at $\gamma_{12}^{F, M}=3-\gamma_{11}^{F, M}$. The combined fraction of high-status individuals in the population increases in all cases. Parameters are as in Figure 2 unless otherwise noted. 


\subsection{Intervention and HIV prevalence}

We now return to our main goal to study the effect of choice disability and related interventions on HIV transmission and prevention. We saw that single-sex choice-disability interventions may have unintended side effects for the choice status of the opposite sex. Unlike in the previous section, we do not have an explicit expression for the steady states of the model. We use numerical simulations to investigate how these side effects may impact HIV transmission and prevalence. In all our simulations, we consistently found a unique, globally stable equilibrium, either disease-free or endemic.

In our numerical experiments, we focus on the effect that choice disability has on disease transmission. With respect to choice status, we fix choicedisability related parameters to represent the two scenarios from the previous section:

S1 Positive choice-disability feedback, where men benefit from an intervention for women: $\gamma_{11}^{F}=0.75$ and $\gamma_{12}^{F}=2.25$.

S2 Negative choice-disability feedback, where men are worse off from an intervention for women: $\gamma_{11}^{F}=2.25$ and $\gamma_{12}^{F}=0.75$.

In both cases, $\gamma_{11}^{M}=0.75$ and $\gamma_{12}^{M}=2.25$.

In our previous study, we reviewed several aspects of how choice disability interacts with HIV transmission and increases infection risk, for example condom use (or not), anal sex, or dry sex [17]. We found that risk can increase by a factor of 10 when at least one partner is choice disabled (please see the section parameter estimation in [17] and references therein). Here, we introduce a susceptibility parameter $C>1$ that measures the increase in transmission risk to a choice disabled partner. To simplify matters for our strategic exploration, we choose the relative increase independent of sex and choice class of the infectious partner, i.e., we set $C=\frac{\beta_{\mathbf{\bullet}_{j}}^{\mathbf{\bullet}}}{\beta_{1_{j}}^{\mathbf{\bullet}}}$ for $j=1,2$ and $\bullet=M, F$.

We still have two cases to consider. In the first, we make the neutral assumption that choice disability increases HIV transmission for men and women in a similar way. According to the above explanation, we choose transmission parameters $\beta_{i j}^{M, F}$ as

$$
\beta^{M}=\left[\begin{array}{cc}
\frac{1.5}{C} & 1.5 \\
1.5 & 1.5 C
\end{array}\right], \quad \beta^{F}=\left[\begin{array}{cc}
\frac{0.5}{C} & 0.5 \\
0.5 & 0.5 C
\end{array}\right] .
$$


We chose the values in $\beta^{F}$ smaller than in $\beta^{M}$ since HIV transmission from women to men is less likely than in the reverse direction [11].

In Figure 4, we plot the changes in HIV prevalence in the different compartments before and after a choice-disability intervention for women only, as described in the previous section. The difference for women

$$
\frac{I_{i}^{F}}{N_{i}^{F}}-\frac{I_{i I}^{F}}{N_{i}^{F} I}
$$

(and similarly defined for men and for the total population) is positive if the intervention (indexed with $I$ ) reduces prevalence, i.e., has the desired outcome. We observe three effects in this scenario.

First, most compartments benefit from an intervention (aimed at women only) in the sense that their HIV prevalence decreases. The only exception to this rule are the compartments of choice-enabled women (top left panel) and choice-enabled people (top right). Intervention increases prevalence in these compartments not because more choice-enabled women become HIV positive but because more HIV-positive women become choice enabled. When we look at the numbers for all women (bottom left panel), we see that HIV prevalence decreases overall.

Secondly, when men indirectly benefit from the intervention (scenario $\mathrm{S} 1$ ), the overall positive effect of the intervention is stronger (diamonds in Figure 4) than when the choice status of men is harmed by the intervention (scenario S2, circles in Figure 4).

Thirdly, the effect is not monotone in $C$. When $C$ is small, the disease transmission rates chosen are so small that the disease-free equilibrium is stable. When the prevalence is zero, the intervention does not make a difference. As $C$ increases, disease transmission is strong enough for the disease to become endemic. Accordingly, prevalence without intervention increases, this initially higher prevalence allows more significant the reductions upon intervention. When $C$ is very high, disease transmission is particularly high in the choice-disabled class so that we observe a saturation effect. The intervention cannot keep up with the rate at which new infections occur in the choice-disable class, and its relative effect decreases.

As we saw in the model for only choice-disability, an interventions to raise women's choice status can have unintended side effects of lowering men's choice status (Section 4.1). In the preceding example of the model 

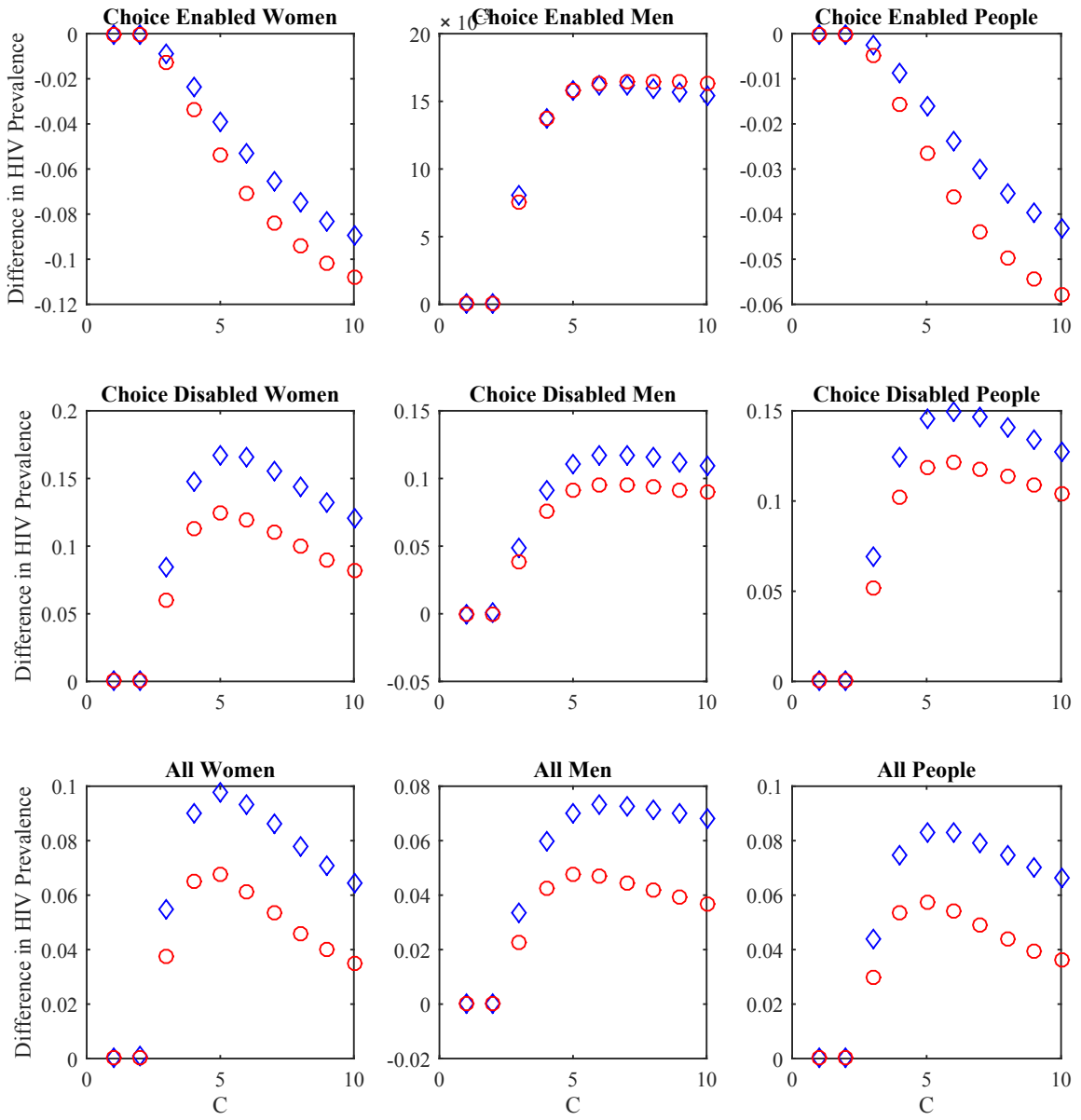

Figure 4: Difference in HIV prevalence (at steady state) after women-only intervention with an equal choice disability effect on transmission for men and women. Diamonds indicate the case where both men and women benefit from the intervention (scenario S1). Circles indicate the case where men are harmed by the intervention (scenario S2). Other parameters are as in previous figures, with transmission rates given by (15). 
for disease and choice-disability, HIV prevalence in men and in the overall population decreased from the intervention, even when the choice status of men decreased. Will intervention always be beneficial or are there cases where the decrease in choice status of men can jeopardize the overall success of the intervention in terms of HIV prevalence?

We construct an example where overall HIV prevalence actually increases from an intervention for women only. We consider the worst case scenario that choice disability has a larger impact on HIV transmission to and from men than for women. In other words, men are more strongly affected by choice disability than women. Correspondingly, we choose transmission parameters as

$$
\beta^{M}=\left[\begin{array}{cc}
\frac{0.6}{C} & (0.6)(10) \\
0.6 & (0.6)(10) C
\end{array}\right], \quad \beta^{F}=\left[\begin{array}{cc}
\frac{0.2}{C} & 0.2 \\
(0.2)(10) & (0.2)(10) C
\end{array}\right] .
$$

The transmission rates from choice-disabled men to all women (second column of the matrix $\beta^{M}$ ) and the rates from all women to choice-disabled men (second row of the matrix $\beta^{F}$ ) are multiplied by a factor of 10 . Parameter $C$ has the same meaning as before; it measures the increase in susceptibility of choice-disabled women to transmission from choice-enabled men and the increase in transmission from choice-disabled women to choice-enabled men. However, the transmission to and from choice disabled men is increased by an additional factor of 10 .

In this case, we vary parameter $C$ only between 1 (where choice disability only affects men) and 2 (where men experience a large choice-disability effect for men but women only a small one). In Figure 5, we see that an intervention aimed at increasing the choice status of women could have a negative effect on HIV prevalence in the total population. As in the previous figure, we observe that prevalence increases among choice-enabled women and people; the reason is the same as before. Contrary to the previous figure, prevalence now also increases (i.e., the difference in prevalence is negative) in all men and all people in the S2 scenario (circles) where men's status is indirectly harmed by the intervention for women through the interaction patterns given by $\gamma_{i j}^{\bullet}$. Hence, unintended side effects can happen at the population level if the intervention has the side effect of reducing the choice status of men and if choice disability in men has a greater impact on HIV transmission than choice disability in women. We discuss the validity and likelihood of occurrence of this effect in the next section. 

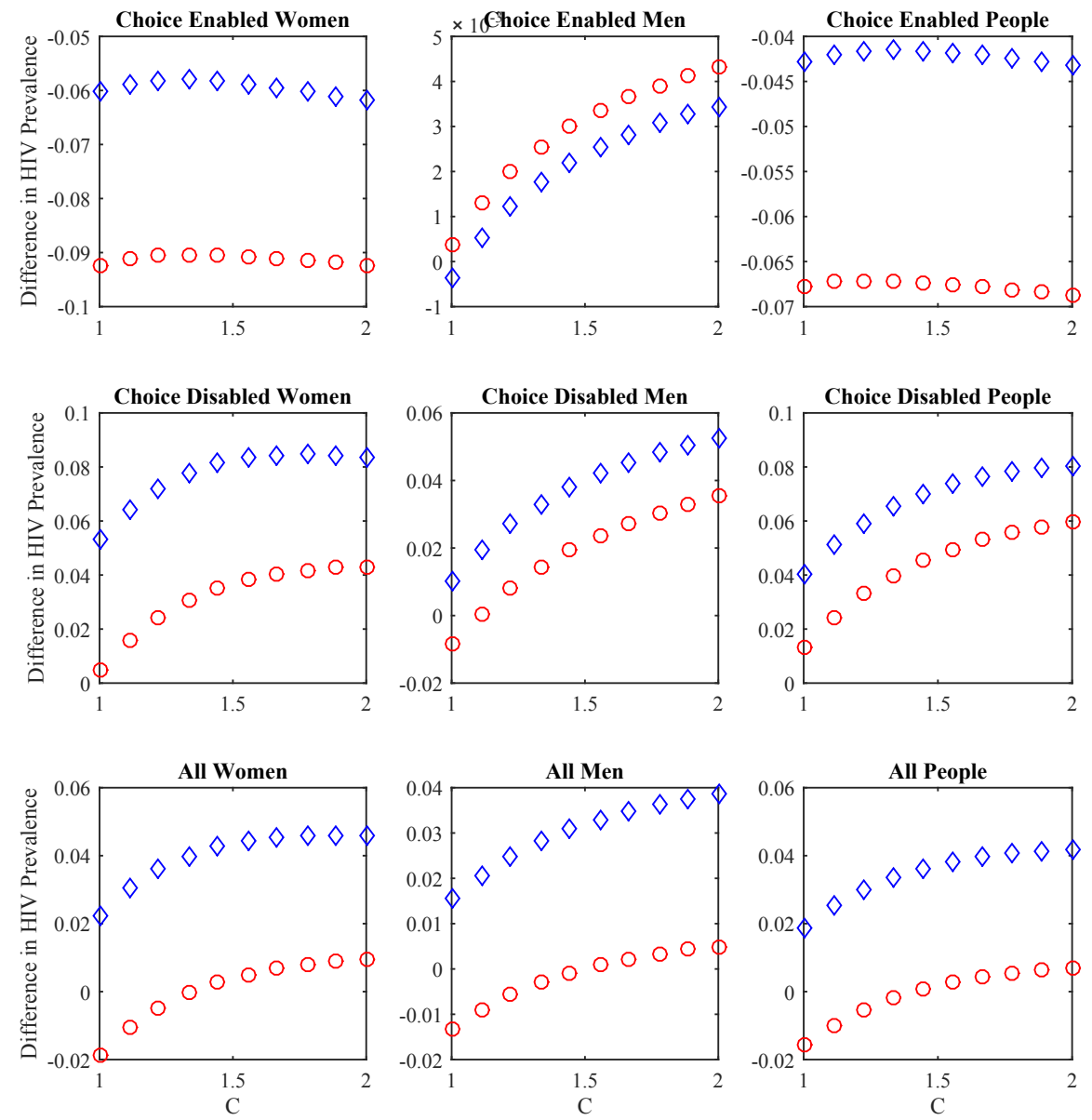

Figure 5: Difference in HIV prevalence (at steady state) after women-only intervention with a greater choice-disability effect on transmission for men than women. Diamonds indicate the case where both men and women benefit from the intervention (scenario S1). Circles indicate the case where men are harmed by the intervention (scenario S2). Other parameters are as in previous figures with transmission rates given by (16). 


\section{Discussion and Conclusions}

Despite enormous financial efforts to intervene against HIV transmission with medical programs, the prevalence of the disease seems to not decline as quickly as desired or even not decline at all in some countries of sub-Saharan Africa [2, 33], please see [32] for an interactive web resource. It has been suggested that the effectiveness of medical intervention is limited by individual "choice disability," the inability of people to implement available protective measures [2, 3]. Most modeling studies of interventions against infectious diseases consider medical interventions, e.g. condom use against STDs [28] or vaccination programs [15]. Some include aspects of individual choice in the form of compliance or non-compliance with mandatory or optional programs [15]; others consider compliance in a game-theoretic framework [8]. None of these models capture the aspect of choice disability where people are unable - for individual or relational reasons - to implement prevention measures for themselves. In our previous work, we considered individual reasons [17]; here, we propose a novel model framework to capture individual and relational aspects of choice disability and their effects on intervention programs. Our analysis focuses on the relational aspects.

The choice-structure of our model is related to the model of social dynamics developed in $[9,10]$. However, their model assumed that social interactions are conservative, for example in economic transactions, which is not the case here. Their and our models are a subclass of generalized kinetic models that describe changes in a (continuous) state variable due to interactions between members of a population [6, 7, 22]. As our model jointly generalizes basic infectious disease models and multi-species Lotka-Volterra models, we expect that a general qualitative theory will be quite complicated, as one can expect oscillatory solutions in general. For carefully constrained bilinear contact terms, we would hope that some of the techniques developed and summarized in [21] can be extended. Our result about the uniqueness and global asymptotic stability of the steady state in the 'choice-disability model' is a first step in this direction, but many open questions remain on the qualitative analysis of the model. Our model applies to contacts of the sexually active portion of the population before they enter stable (monogamous) relationships, and our results are about steady states and their dependence on parameters. In other situations, disease transmission terms other than bilinear mass-action could be more appropriate.

The distinction between individual and relational factors of choice disabil- 
ity is not always clear nor easy. Risk factors such as income or education are individual/absolute factors that determine various options and behaviors of a person, e.g. whether they can afford and properly use condoms. But income and education are also relative factors in that income/education disparity within a relation may lead to choice disability [2]. For example, a woman who knows about infection and protection may not be able to negotiate condom use in a relationship with an uneducated partner, in particular when social norms of male dominance are prevalent. Obviously, intimate partner violence, rape or sexual assault all occur in relationships between at least two people and can severely limit people's choices [3]. Clearly, experiences of rape or sexual assault remain with the person who experienced them and get carried into potential future relationships. In particular, if such violence occurs in the relationship between two choice-enabled partners, at least one of them will carry a choice-disability factor from then on. But even factors such as extreme poverty and food insufficiency, which are typically considered individual factors, can have relational roots and consequences. For example, if one person suffers from extreme poverty and falls severely ill as a consequence, their partner might slide into poverty while caring for them, and even lose their job and thereby end up in poverty and choice disabled as well. Our model can incorporate individual and relational aspects via individual rates $\lambda_{i}^{\bullet}>0$ and contact rates $\gamma_{i j}^{\bullet}$. Our previous work was on individual factors [17]; our main focus here is on relational factors and their potential to incur indirect side effects of intervention (see more details below).

Many observations and empirical studies show that women bear a much larger share of choice disability and its negative consequences, such as the inability of HIV prevention [2, 3]. Consequently, women are often the focus group for structural (i.e., non-medical) intervention $[5,13,18]$, and most rightly so. When resources are focused on one group, other groups in need (e.g. choice-disabled men) may suffer in that they may not receive the resources needed, so that resource allocation can pose serious moral and practical dilemmas [26]. What our model shows in addition is that indirect side effects could prevent the focus group from optimally benefiting from the intervention and negatively affect other groups. In the worst case, indirect side effects could make the entire population worse off. We do not believe that such indirect negative effects are common or unavoidable. In particular, we do not want our results to be construed as justification for not implementing support programs for women. But we can think of examples where indirect negative effects may arise, and we want to point out the mechanisms behind. 
We give an example scenario in the next paragraph.

We saw that the overall effect of a choice-disability intervention depends greatly on the contact coefficients that encode the culture around relationships and the ways in which power gradients may form within them. Social norms in many cultures see men as the stronger partner who make decisions for the couple. Fortunately, as women obtain equal and higher levels of education, these norms are being challenged. Parameters $\gamma_{11}^{M}$ and $\gamma_{11}^{F}$ indicate the extent to which choice-enabled men and women deny their partners choices. This denial may occur through exploitative behaviors, such as transactional sex or intimate partner violence, but may also manifest in other ways such as resisting condom use. Men may benefit from an intervention targeting women if choice-enabled women are less likely to restrict their parters' choices than choice-disabled women. This scenario could arise when choice-disabled women reduce the choices of their male partners through the use of economic resources, intimate partner violence or others. Men are harmed by the intervention if choice-enabled women are more likely to restrict their partners' choices than choice disabled women. This scenario can arise when choiceenabled women engage in exploitative behavior such as transactional sex. For example, women with higher income than their partners are more likely to have multiple sexual partners than women with less income than their partners [20]. As mentioned above, we have currently no way to estimate the impact of so-called 'sugar mommies' and 'rent boys' on the transitions between choice enabled and choice disabled compartments. But we know that the effects are visible, and our model says that if they are strong, they can limit the success of well-meant programs.

So far, we focused on acquiring choice-disability. Recovering from it, through intervention programs, was modeled as a linear term. Any intervention program has to trade-off the number of people that it can reach with the amount of time and money it can sped on each person. The single-sex intervention used in the simulations in Section 4 would require raising the status of approximately $6.7 \%$ of the low-status women each year. As the low-status population is only a fraction of the total population of women, the actual size of the intervention could be even smaller, provided it could be targeted effectively. Whether one should target all choice-disabled women or only those who are HIV positive is an open question that will be important to answer once sufficiently reliable values for other parameters have been found.

Probably more exciting is the prospect that not only the acquisition but also the recovery from choice disability could have a relational component. 
Our model allows for such intervention effects through parameters $\psi_{i j}^{\bullet}$. Not much in known about the relational acquisition parameters $\gamma_{i j}^{\bullet}$, but even less about the recovery parameters, which is why we did not explore the effects of these parameters in our analysis. However, this knowledge gap could be filled soon. Recent and ongoing trials aim to reduce HIV infections by targeting choice disability $[5,13]$. They use multiple interventions: a universal intervention, targeting men and women to become choice enabled; a single-sex intervention, targeting young, choice-disabled women; and an institutional intervention that aims to raise awareness of choice disability in existing HIV prevention programs. Another intervention, the Stepping Stones program, targets young men and women and uses a participatory learning approach to teach about a variety of topics related to sexual health [24]. Several of the topics included in the program such as gender violence are also factors in choice disability. Many of the areas in which this intervention saw improvement, such as perpetration of intimate partner violence and participation in transactional sex by men, are also related to choice disability. We are looking forward to incorporating these results into our model.

Despite all empirical studies, contact coefficients for choice-disability acquisition and HIV transmission are difficult to measure, and without these parameters it is difficult to know in advance how a single-sex intervention will impact the opposite sex. However, in most scenarios we observed substantial reductions in overall HIV prevalence. Furthermore, the cases in which we observe a negative outcome for HIV prevalence due to interventions are probably unrealistic. Negative outcomes occurred when men lost status due to the intervention targeting women and were more heavily impacted by choice disability than women. In reality the opposite is thought to be the case as HIV prevalence increases drastically for women with even low levels of choice disability [2]. While single-sex interventions have the potential for unintended side effects, these seem unlikely to include an increase in HIV prevalence.

Overall, further work should be done to determine realistic parameter values for the particular contexts in which choice disability interventions are to be implemented since these types of interventions could have a significant impact on HIV prevalence. In any given scenario, the binary structure in our application may need to be extended to include more (choice-) status classes as in the general model (1). Choice disability should be considered as a important factor in a comprehensive HIV prevention strategy. 


\section{Acknowledgments}

The authors thank Neil Andersson for inspiration and discussions. We also thank two anonymous reviewers for their detailed and insightful comments that greatly improved our work. RdB gratefully acknowledges funding from CIET Canada (Community Information, Empowerment and Transparency, see www.ciet.org). FL is funded by a Discovery Grant from the Natural Science and Engineering Research Council of Canada (NSERC, grant number RGPIN-2016-0495).

\section{References}

[1] R.D. Anderson and G.P. Garnett. Mathematical models of the transmission and control of sexually transmitted diseases. Sexually transmitted diseases, 27:636-643, 2000.

[2] N. Andersson and A. Cockcroft. Choice-disability and hiv infection: a cross sectional study of HIV status in Botswana, Namibia and Swaziland. AIDS and Behavior, 16(1):189-198, 2012.

[3] N. Andersson, A. Cockcroft, and B. Shea. Gender-based violence and HIV: relevance for HIV prevention in hyperendemic countries of southern Africa. AIDS, 22:S73-S86, 2008.

[4] N. Andersson, S. Paredes-Solís, D. Milne, K. Omer, N. Marokoane, D. Laetsang, and A. Cockcroft. Prevalence and risk factors for forced or coerced sex among school-going youth: national cross-sectional studies in 10 southern African countries in 2003 and 2007. BMJ Open, 2(2): e000754, 2012.

[5] N. Andersson, A. Cockcroft, L. Thabane, N. Marokoane, D. Laetsang, and M. Masisi. HIV prevention in favour of the choice-disabled in southern Africa: study protocol for a randomised controlled trial. Trials, 14 (1):274, 2013.

[6] L. Arlotti, N. Bellomo, and K. Latrach. From the Jager and Segel model to kinetic population dynamics nonlinear evolution problems and applications. Mathematical and Computer Modelling, 30(1):15-40, 1999. 
[7] L. Arlotti, N. Bellomo, and E. De Angelis. Generalized kinetic (Boltzmann) models: mathematical structures and applications. Mathematical Models and Methods in Applied Sciences, 12(04):567-591, 2002.

[8] C.T. Bauch and D.J.D. Earn. Vaccination and the theory of games. Proc Nat Acad Sci, 101(36):13391-13394, 2004.

[9] M.L. Bertotti and M. Delitala. From discrete kinetic and stochastic game theory to modelling complex systems. Mathematical Models and Methods in Applied Sciences, 14(7):1061-1084, 2004.

[10] M.L. Bertotti and M. Delitala. Conservation laws and asymptotic behavior of a model of social dynamics. Nonlinear Analysis: Real World Applications, 9(1):183-196, 2008.

[11] M.-C. Boily, R.F. Baggaley, L. Wang, Benoit Masse, R.G. White, R.J. Hayes, and M. Alary. Heterosexual risk of hiv-1 infection per sexual act: systematic review and meta-analysis of observational studies. The Lancet, 9:118-129, 2009.

[12] F. Brauer and C. Castillo-Chavez. Mathematical models for communicable diseases. SIAM, 2012.

[13] M. Cameron, A. Cockcroft, G.W. Waichigo, N. Marokoane, D. Laetsang, and N. Andersson. From knowledge to action: participant stories of a population health intervention to reduce gender violence and HIV in three southern African countries. AIDS Care, 26(12):1534-1540, 2014.

[14] A. Cockcroft, J.L. Kunda, L. Kgakole, M. Masisi, D. Laetsang, A. HoFoster, N. Marokoane, and N. Andersson. Community views of intergenerational sex: Findings from focus groups in Botswana, Namibia and Swaziland. Psychology, Health $\& 3$ Medicine, 15(5):507-514, 2010.

[15] E.J. Dasbach, E.H. Elbasha, and R.P. Insinga. Mathematical models for predicting the epidemiologic and economic impact of vaccination against human papillomavirus infection and disease. Epidemiologic Reviews, 28: 88-100, 2006.

[16] R. deBoer. Estimating The Undiagnosed HIV-Positive Population A Mathematical Modeling Study. PhD thesis, 2014. 
[17] R. deBoer, J. Musgrave, N. Anderson, and F. Lutscher. The importance of choice disability and structural intervention in the HIV epidemic in sub-Saharan Africa. PLoS ONE, 12(4):e0175297, 2017.

[18] D. Hallfors, H. Cho, S. Rusakaniko, B. Iritani, J. Mapfumo, and C. Halpern. Supporting adolescent orphan girls to stay in school as hiv risk prevention: evidence from a randomized controlled trial in zimbabwe. American Journal of Public Health, 101:1082-1088, 2011.

[19] H.W. Hethcote and J.W. Van Ark. Modeling HIV transmission and AIDS in the United States. Springer, 1992.

[20] A. Ho-Foster, D. Laetsang, M. Masisi, M. Anderson, D. Thoiwe, A. Cockcroft, and N. Andersson. Gender-specific patterns of multiple concurrent sexual partnerships: a national cross sectional survey in Botswana. AIDS Care, 22(8):1006-1011, 2010.

[21] J. Hofbauer and K. Sigmund. Evolutionary games and population dynamics. Cambridge University Press, 1998.

[22] E. Jäger and L.A. Segel. On the distribution of dominance in populations of social organisms. SIAM Journal on Applied Mathematics, 52(5):14421468, 1992.

[23] R. Jewkes. Transactional sex and HIV incidence in a cohort of young women in the Stepping Stones Trial. Journal of AIDS \& Clinical Research, 2012.

[24] R. Jewkes, M. Nduna, J. Levin, N. Jama, K. Dunkle, A. Puren, N. Duvvury, et al. Impact of stepping stones on incidence of HIV and HSV-2 and sexual behaviour in rural South Africa: cluster randomised controlled trial. BMJ, 337:a506, 2008.

[25] R.K. Jewkes, K. Dunkle, M. Nduna, and N. Shai. Intimate partner violence, relationship power inequity, and incidence of HIV infection in young women in South Africa: a cohort study. The Lancet, 376(9734): 41-48, 2010.

[26] A. Lasry, A. Richter, and F. Lutscher. Recommendations for increasing the use of HIV/AIDS resource allocation models. BMC Public Health, 9 (suppl 1):S8, 2009. 
[27] S. Leclerc-Madlala. Age-disparate and intergenerational sex in southern Africa: the dynamics of hypervulnerability. AIDS, 22:S17-S25, 2008.

[28] N. Malunguza, S. Mushayabasa, C. Chiyaka, and Z. Mukandavire. Reproduction numbers and sub-threshold endemic equilibria for compartmental models of disease transmission. Computational and Mathematical Methods in Medicine, 11:201-222, 2010.

[29] C. McCluskey. Using Lyapunov functions to construct Lyapunov functionals for delay differential equations. SIAM J Applied Dynamical Systems, 14:1-24, 2015.

[30] N.J.D. Nagelkerke, P. Jha, S.J. de Vlas, E.L. Korenromp, S. Moses, J.F. Blanchard, and F.A. Plummer. Modelling HIV/AIDS epidemics in botswana and india: impact of interventions to prevent transmission. Bulletin of the World Health Organization, 80:89-96, 2002.

[31] A.S. Perelson and M.R. Ribeiro. Modeling the within-host dynamics of HIV infection. BMC Biology, 11:96, 2013.

[32] UNAIDS. Aidsinfo. website accessed Sept. 7, 2016. http://aidsinfo. unaids.org/.

[33] UNAIDS. Prevention gap report, 2016. Joint United Nations Programme on HIV/AIDS.

[34] J. Watmough and P. van den Driessche. Reproduction numbers and sub-threshold endemic equilibria for compartmental models of disease transmission. Mathematical Biosciences, 180:29-48, 2002. 